\title{
Synovial fluid chondroitin sulphate epitopes 3B3 and 7D4, and glycosaminoglycan in human knee osteoarthritis after exercise
}

\author{
Judith C Bautch, Murray K Clayton, Qili Chu, Kenneth A Johnson
}

Winona State

University, Winona,

Minnesota, USA

J C Bautch

Department of Statistics and Plant Pathology, University of Wisconsin-Madison, Madison, Wisconsin, USA

M K Clayton

Department of Surgical Sciences, University of Wisconsin-Madison Q Chu

Department of Veterinary Clinical Sciences, The Ohio State University, Columbus, Ohio, USA K A Johnson

Correspondence to: Professor Kenneth A Johnson, The Ohio State University, Department of Veterinary Clinical Sciences, 601 Vernon L Tharp Street, Columbus Ohio 43210-1089, USA Email:

johnson.2064@osu.edu

Accepted for publication 10 April 2000

\begin{abstract}
Objective-Walking exercise alleviates some symptoms, such as pain, in patients with mild to moderate knee osteoarthritis (OA). However, a major concern is that weightbearing exercise on osteoarthritic joints may exacerbate articular cartilage degradation. Loading of proteoglycan depleted articular cartilage in vitro increased expression of the chondroitin sulphate epitope $3 B 3$, suggesting that loading may influence metabolism of osteoarthritic cartilage. This study aimed at evaluating the effects of walking exercise on articular cartilage metabolism in patients with knee $O A$, as reflected by changes in concentrations of synovial fluid markers.
\end{abstract}

Methods-Thirty elderly patients with knee OA (Kellgren-Lawrence grades II to IV) were randomly allocated to control (n $=15)$ and 12 week exercise $(n=15)$ groups. Synovial fluid obtained from 21 of the patients at time zero and after 12 weeks was examined by enzyme linked immunosorbent assay (ELISA) for the chondroitin sulphate epitopes $3 \mathrm{~B} 3$ and 7D4, and by a dye binding assay with 1,9-dimethylmethylene blue for total sulphated glycosaminoglycan (GAG) concentrations. The 3B3/GAG and 7D4/GAG ratios were calculated.

Results-No significant changes in concentrations of $3 \mathrm{~B} 3,7 \mathrm{D} 4, \mathrm{GAG}, 3 \mathrm{~B} 3 / \mathrm{GAG}$, or 7D4/GAG between time zero and 12 weeks were found in either group. However, there were significant declines in $3 B 3(p=0.001)$, GAG $(p=0.007)$, and the $3 B 3 / G A G$ ratio $(p=0.049)$ with aging.

Conclusion-Twelve weeks of walking exercise had no demonstrable adverse effects on articular cartilage metabolism, as reflected by the concentrations of synovial fluid GAG or the chondroitin sulphate epitopes 3B3 and 7D4.

(Ann Rheum Dis 2000;59:887-891)

Mild to moderate osteoarthritis (OA) of the knee has a prevalence of about $65 \%$ in the middle aged and elderly population, especially women, though progression to severe disease is uncommon. ${ }^{12}$ Recommendations for management of mild knee OA include control of obesity, quadriceps strengthening exercise, and walking exercise. ${ }^{13}$ Although walking exercise alleviates some symptoms of knee OA, including pain,,$^{5-7}$ one concern is that weightbearing activity may exacerbate articular cartilage degradation.

Joint loading at physiological levels is necessary for maintenance of normal joint morphology, but overuse may be damaging. Prolonged running exercise by experimental dogs resulted in alterations in stiffness, morphology, and proteoglycan content of knee articular cartilage, ${ }^{8-12}$ and horses engaged in strenuous exercise had reduced aggrecan and decorin synthesis in carpal articular cartilage. ${ }^{13}$ Furthermore, female athletes engaged in weightbearing sports (competitive running and tennis) had an increased risk of developing radiological signs of hip and knee $\mathrm{OA}$ in later life. ${ }^{14}$ Moreover, immediately after a single bout of exercise (running or soccer) by healthy subjects, levels of synovial fluid markers of cartilage matrix degradation were raised. ${ }^{15}$ Although these data suggest that excessive weightbearing exercise may have damaging effects on normal joints, little is known about effects of weightbearing exercise on osteoarthritic joints.

Previously, we found that keratan sulphate concentrations in synovial fluid in osteoarthritic knees of elderly patients were unaltered by a 12 week exercise programme. ${ }^{5}$ By contrast, keratan sulphate concentrations in synovial fluid of equine carpal joints with acute osteochondral defects and secondary OA, were raised significantly after 17 weeks of exercise. ${ }^{16}$ This apparent disparity in findings might have been due to differences in chronicity of disease. In chronic OA, concentrations of keratan sulphate in synovial fluid are low, and unaltered by episodes of acute inflammation. ${ }^{17}$ Some other proposed markers of altered cartilage metabolism in OA are epitopes on chondroitin sulphate chains recognised by the monoclonal antibodies 3B3 and 7D4. ${ }^{18}{ }^{19}$ Articular cartilage from osteoarthritic human knees had increased expression of these markers. ${ }^{20}$ Also, levels of 3B3 and 7D4 in synovial fluid were raised in human knees with acute traumatic cruciate ligament and meniscal injuries. ${ }^{21}$ Intermittent compression of bovine articular cartilage in vitro, using loading regimens within physiological range and frequency, increased the expression of $3 \mathrm{~B} 3 .^{22}$ This effect was more pronounced in proteoglycan-depleted cartilage, suggesting that loading may be harmful to cartilage that has pre-existing matrix damage. ${ }^{23}$ To examine further the effects of weightbearing exercise on osteoarthritic joints in vivo, we measured 3B3 and 7D4 concentrations in synovial fluid from osteoarthritic joints of 
Table 1 Demographics and characteristics of subjects with knee osteoarthritis (mean (SEM))

\begin{tabular}{lll}
\hline & Control $(n=11)$ & Exercise $(n=10)$ \\
\hline Male:female & $4: 7$ & $3: 7$ \\
Age & $73.1(1.9)$ & $66.3(1.9)^{\star}$ \\
Body mass index & $26.4(0.7)$ & $30.8(1.3) \dagger$ \\
Kellgren-Lawrence radiographic grade & \\
II & 4 & 2 \\
III & 2 & 4 \\
IV & 5 & 4 \\
\hline
\end{tabular}

$\star$ Difference between groups significant $(p=0.015)$.

$\dagger$ Difference between groups significant $(\mathrm{p}=0.006)$.

patients undergoing a 12 week walking exercise programme. We proposed the hypothesis that increased mechanical loading of osteoarthritic articular cartilage would result in increased concentrations of 3B3 and 7D4 in the synovial fluid.

\section{Methods}

SUBJECTS

With approval of the institutional ethics committee, subjects were recruited to the study as described previously. ${ }^{5}$ In brief, they were at least 58 years of age, and were diagnosed with stable OA, which met clinical and radiographic criteria for primary knee OA. ${ }^{24}$ Severity of knee OA was graded radiographically by the Kellgren-Lawrence (K-L) criteria. ${ }^{25}$ Most subjects had asymmetric bilateral disease and the knee with the highest radiographic grade was selected as the "study knee". Body mass index (BMI) was calculated according to standard criteria. ${ }^{26}$ Subjects had no physical or medical problems for which participation in an exercise programme would have been contraindicated. They were not currently enrolled in a regular exercise programme and had not received any intra-articular or systemic glucocorticoid drug treatment within the preceding two years. Most of the subjects periodically used various analgesics for knee pain, but none routinely used any non-steroidal anti-inflammatory drugs with known potential for significantly altering cartilage metabolism.

\section{EXERCISE PROGRAMME}

After informed consent was obtained, subjects were randomly allocated to two groups: an exercise group $(n=15)$ and a control group $(n=15)$. The exercise programme has been previously described. ${ }^{5}$ Briefly, the exercise group participated in a weekly health education session, and a one hour exercise session three times a week, which included quadriceps strengthening exercises and an individualised

Table 2 p Values derived from analysis of synovial fluid data using split plot model for group, time, and group by time interaction, and backward elimination to determine significance of covariates (body mass index, sex, age, and Kellgren-Lawrence grade)

\begin{tabular}{llllll}
\hline & $\log 3 B 3$ & $\log 7 D 4$ & $\log G A G \dagger$ & $3 B 3 / G A G$ & $7 D 4 / G A G$ \\
\hline Group (control or exercise) & $0.049^{\star}$ & 0.829 & $0.009^{\star}$ & 0.404 & 0.320 \\
Time & 0.409 & 0.161 & 0.841 & 0.549 & 0.192 \\
Group $\times$ time interaction & 0.203 & 0.789 & 0.057 & 0.118 & 0.302 \\
Body mass index & $\mathrm{x}$ & $\mathrm{x}$ & $\mathrm{x}$ & $\mathrm{x}$ & $\mathrm{x}$ \\
Sex & $\mathrm{x}$ & $\mathrm{x}$ & $\mathrm{x}$ & $\mathrm{x}$ & $\mathrm{x}$ \\
Age & $0.001^{\star}$ & $\mathrm{x}$ & $0.007^{\star}$ & $0.049^{\star}$ & $\mathrm{x}$ \\
Kellgren-Lawrence grade & $\mathrm{x}$ & $\mathrm{x}$ & $\mathrm{x}$ & $\mathrm{x}$ & $\mathrm{x}$ \\
\hline
\end{tabular}

$\star$ Significant effect $(\mathrm{p}<0.05)$.

†GAG = glycosaminoglycan

$\mathrm{x}$ corresponds to covariates that were removed as a result of backward elimination. low intensity walking prescription. The control group was a delayed treatment group, and had a weekly health education class. Control subjects were instructed not to alter their routine pre-study activity levels. The duration of the study was 12 weeks.

ARTHROCENTESIS AND SYNOVIAL FLUID PREPARATION

Synovial fluid was obtained from the knee joints by arthrocentesis before and within 24 hours of completion of the intervention phase of the study. An aliquot was immediately examined microscopically, and in all samples leucocyte counts were less than $10^{9} / 1$ and crystals were not seen under polarised illumination. The remaining fluid was centrifuged at 10000 $\mathrm{rpm}$ and the supernatant decanted, split into aliquots, and stored at $-70^{\circ} \mathrm{C}$.

\section{SULPHATED GLYCOSAMINOGLYCAN CONTENT OF} SYNOVIAL FLUID

Aliquots of synovial fluid supernatant were digested in buffered papain solution (20 units/mg protein, Sigma Chemical Co, St Louis, MO, USA) as described previously. ${ }^{21}$ After inactivation of the enzyme by addition of iodoacetic acid, supernatants were treated with hyaluronidase (Streptomyces hyalurolyticus, 2000 TRU/mg; Seikaguka Corp, Japan), and then sulphated glycosaminoglycan (GAG) levels were measured using a dye binding assay. ${ }^{1721}{ }^{27}$ Shark chondroitin sulphate standards (Sigma Chemical Co, St Louis, MO, USA) in the range $0-40 \mu \mathrm{l} / \mathrm{ml}$ were prepared. After addition of 1,9-dimethylmethylene blue (Molecular Probes, Inc, USA) solution ${ }^{17}{ }^{27}$ to each plate well (Immulon 2, Dynatech Lab, Inc, USA) containing sample or standard, shifts in absorbency were detected immediately at $530 \mathrm{~nm}$ on a plate reader (EL 312e Bio-Kinetics Microplate Reader, Bio-Tek Instruments, VT, USA).

\section{B3 ASSAY}

A competitive equilibrium ELISA based on a previously described method ${ }^{21}$ was used with the following variations to quantify $3 \mathrm{~B} 3$ epitope levels in synovial fluid. Synovial fluid samples were diluted 1:10 in saline. Standards of porcine aggrecan core protein (a generous gift from Professor Michael T Bayliss, London) in the range $1.95-500 \mathrm{ng} / \mathrm{ml}$ were prepared, and monoclonal antibody 3B3 (Seikaguka Co, Tokyo, Japan) at a dilution of 1:1600 (final dilution 1:3200) was used. Blank (Tris buffer only) and reference wells (antibody only, no competing antigen) were also included in each plate. The second antibody, antimouse $\operatorname{IgM}$ peroxidase conjugate (Sigma Chemical Co, St Louis, MO, USA), was diluted 1:1000. Colour reaction was read 60 minutes after addition of peroxidase substrate (ABTS, Sigma) on a plate reader at $405 \mathrm{~nm}$. The $3 \mathrm{~B} 3$ levels were calculated in equivalent weight of the antigen standard, using the absorbency compared with reference wells (no antigen), and by reading this value against the standard curve of percentage of inhibition against the standard antigen concentration. ${ }^{21}$ 

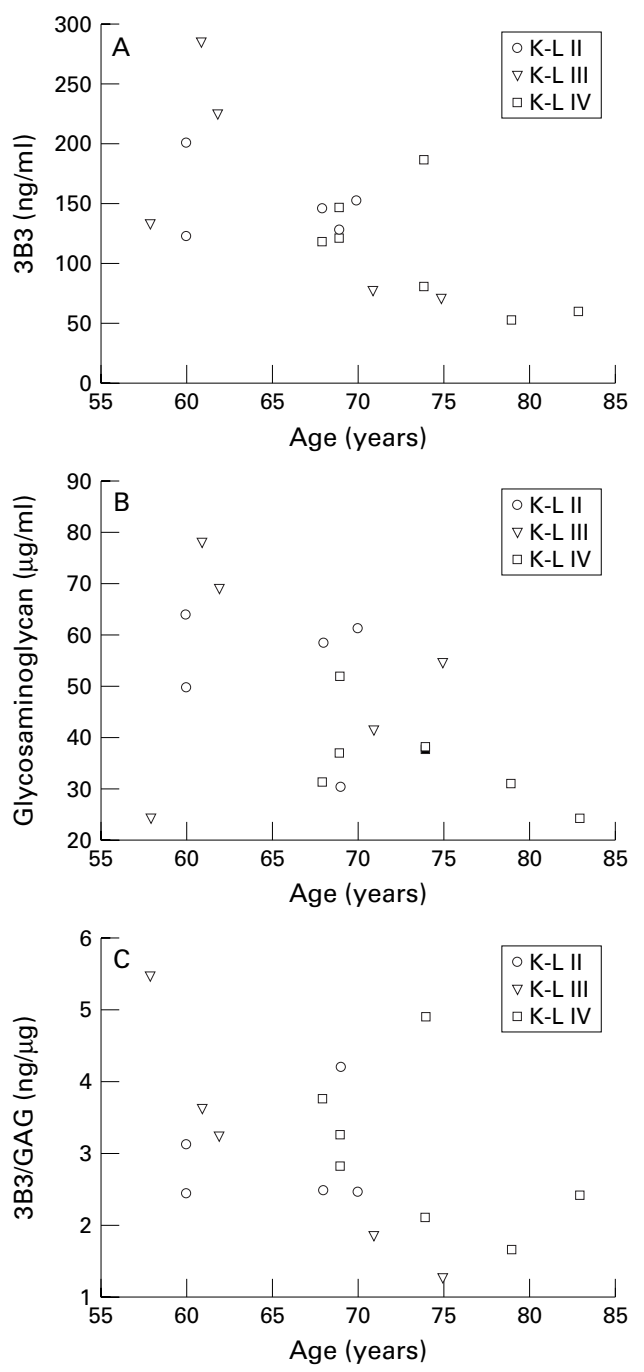

Figure 1 Relation between patient age and levels of $(A)$ $3 B 3,(B)$ glycosaminoglycan $(G A G)$, and $(C)$ the $3 B 3 / G A G$ ratio in knee joint synovial fluid samples obtained at time zero, before the start of the study. The Kellgren-Lawrence $(K-L)$ radiographic grade of osteoarthritis for each joint is also indicated.

7D4 ASSAY

This assay was similar to the 3B3 ELISA, with the following exceptions. Monoclonal antibody 7D4 was a generous gift of Professor Bruce Caterson (University of Cardiff). The antigen used for coating microplates and preparation of standards was bovine laryngeal cartilage proteo-

Table 3 Synovial fluid 3B3, 7D4, and glycosaminoglycan (GAG) concentrations and ratios in human knee osteoarthritis after exercise (mean (SEM)) $\dagger$

\begin{tabular}{llll}
\hline & $\begin{array}{l}\text { Time } \\
\text { (weeks) }\end{array}$ & Control $(n=11)$ & Exercise $(n=10)$ \\
\hline Samples analysed & 0 & 9 & 8 \\
& 12 & 7 & 5 \\
$3 \mathrm{~B} 3(\mathrm{ng} / \mathrm{ml})$ & 0 & $124.26(24.2)$ & $147.76(17.8)$ \\
$7 \mathrm{D} 4(\mu \mathrm{g} / \mathrm{ml})$ & 12 & $115.87(21.2)$ & $111.76(25.4)$ \\
& 0 & $62.95(11.5)$ & $90.24(37.2)$ \\
$\mathrm{GAG}(\mu \mathrm{g} / \mathrm{ml})$ & 12 & $55.71(8.3)$ & $27.23(6.5)$ \\
& 0 & $47.66(5.9)$ & $44.35(5.5)$ \\
$3 \mathrm{~B} 3 / \mathrm{GAG}$ ratio $(\mathrm{ng} / \mu \mathrm{g})$ & 12 & $48.97(7.5)^{\star}$ & $32.84(4.9)^{\star}$ \\
$7 \mathrm{D} 4 / \mathrm{GAG}$ ratio $(\mu \mathrm{g} / \mu \mathrm{g})$ & 0 & $2.57(0.3)$ & $3.51(0.4)$ \\
& 12 & $2.39(0.4)$ & $3.44(0.9)$ \\
& 0 & $1.26(0.1)$ & $1.76(0.5)$ \\
& 12 & $1.16(0.2)$ & $0.79(0.1)$ \\
\hline
\end{tabular}

*At 12 weeks, difference between two groups significant $(p=0.002)$.

†Split plot analyses performed on log transformed data but only the non-transformed data are shown here. glycan, generously provided by Professor Michael T Bayliss. Plates were coated at 1 $\mu \mathrm{g} / \mathrm{ml}$ and standards ranged from 0.0156 to 4 $\mu \mathrm{g} / \mathrm{ml}$ of sulphated $\mathrm{GAG}$, as determined by the GAG dye binding assay. Synovial fluid samples were diluted 1:20 in saline. The 7D4 was applied at a dilution of 1:25 000 (final dilution 1:50 000). The second antibody $\operatorname{IgM}$ was diluted 1:1000. Plates were read at two hours after addition of peroxidase substrate (ABTS) on the plate reader.

\section{DATA ANALYSIS}

Age and BMI of subjects in the two groups (control and exercise) were checked to ensure normal distribution, and were compared by unpaired $t$ tests. Data for each of the variables (3B3, 7D4, GAG, 3B3/GAG ratio, and 7D4/ GAG ratio) were analysed using split plot analyses with the "mixed" procedure of SAS (SAS, Cary, NC, USA), which allowed for missing data. For each of the responses a split plot model was fitted where treatment (control or exercise) was the whole plot factor and time (zero or 12 weeks) was the subplot factor; a time by treatment interaction was also included in the model. In addition, to account for possible differences among subjects, sex, age, BMI, and $\mathrm{K}-\mathrm{L}$ grade were included as covariates. Backward elimination was used to remove those covariates that did not contribute significantly to the model. Because some data were not normally distributed, all raw data were log transformed (base 10) and analyses were repeated. The "mixed" procedure was also used to construct $t$ tests to compare group means. Values of $\mathrm{p}<0.05$ were considered significant. When a major result was not significant, a power calculation was performed to estimate the sample size that would be needed to show a significant difference $(\mathrm{p}<0.05) 80 \%$ of the time.

\section{Results}

SUBJECTS

All 30 subjects completed the study, but synovial fluid could only be obtained from knee joints of 21 of these 30 . Therefore observations and analyses for our study were restricted to this subgroup whose characteristics and demographics are summarised in table 1. There was a preponderance of female subjects in the study $(67 \%)$. The control group was significantly older, and had a lower BMI than the exercise group ( $p=0.015$ and $p=0.006$, respectively). The control group was in the category of overweight and the exercise group was obese. ${ }^{26}$

SYNOVIAL FLUID

In the backward elimination the only significant covariate was age for the variables $3 \mathrm{~B} 3$ concentration $(p=0.001)$, GAG concentration $(\mathrm{p}=0.007)$, and $3 \mathrm{~B} 3 / \mathrm{GAG}$ ratio $(\mathrm{p}=0.049)$ (table 2, fig 1). There were no significant changes in concentrations of 3B3, 7D4, GAG, $3 \mathrm{~B} 3 / \mathrm{GAG}$, or $7 \mathrm{D} 4 / \mathrm{GAG}$ between time zero and 12 weeks in either group (table 3). From power calculations it was estimated that for the observed differences to be significant with a power of $80 \%$ in our study, then sample sizes 
for each group of 59 (3B3), 47 (7D4), 10 (GAG), 259 (3B3/GAG), and 11 (7D4/GAG) would be needed. At 12 weeks the mean (SEM) concentration of GAG in the exercise group (32.84 (4.9) $\mu \mathrm{g} / \mathrm{ml})$ was significantly less than in the control group (48.97 (7.5) $\mu \mathrm{g} / \mathrm{ml}$ ) $(p=0.002)$ (table 3$)$. None of the other differences between groups were significant.

\section{Discussion}

Exercise is beneficial in the symptomatic treatment of knee OA, but there is concern about its effect on osteoarthritic cartilage. In our previously reported study of this group of subjects we found that visual analogue scores for pain were significantly reduced in patients undergoing a controlled exercise programme. ${ }^{5}$ One problem with longitudinal clinical studies of $\mathrm{OA}$ is to quantify subtle alterations in progression of articular cartilage breakdown. Direct visualisation of articular cartilage by arthroscopy and magnetic resonance imaging is invasive or expensive. Thus there is considerable interest in attempting to define the use of synovial fluid "markers", such as 3B3 and 7D4, as indirect indicators of articular cartilage metabolism in OA. Antibody 3B3 recognises an epitope on chondroitin sulphate chains that have a non-reducing termination of GlcA $\beta 1$, 3GalNAc6S- ${ }^{28}$ The proportion of such terminal disaccharides, at about $9 \%$, remains constant throughout life, but the immunoreactivity of the proteoglycan varies with the stage of development, maturity, and pathology of connective tissues, because chain length and presentation on solid phase are critical factors for recognition of chain terminations by $3 \mathrm{~B} 3 .^{28}$ Proteoglycans isolated from osteoarthritic cartilage and synovial fluid showed increased immunoreactivity to $3 \mathrm{~B} 3,{ }^{19-21}$ which may, in part, have been due to alterations in the sulphation and length of chains ending with the disaccharide containing the 3B3 epitope. ${ }^{29}$ The epitope for antibody 7D4 is less well characterised, but it appears to recognise subtle combinations of sulphated and non-sulphated disaccharide isomers within the native chondroitin sulphate chain. ${ }^{19}$ Expression of the 7D4 epitope is also more prevalent in proteoglycans extracted from osteoarthritic cartilage and synovial fluid. ${ }^{19}{ }^{30}$ It has been proposed that these are "anabolic" markers of cartilage turnover in OA, and result from attempts to repair or remodel damaged cartilage ${ }^{19}$ By contrast, "catabolic" markers such as BC-3, which is specific for aggrecanase cleavage in the interglobular domain, are indicative of matrix degradation. ${ }^{19}$

Expression of $3 \mathrm{~B} 3$ in both normal and proteoglycan depleted articular cartilage was increased by intermittent loading in vitro, suggesting that weightbearing exercise may be harmful to joints. ${ }^{22}{ }^{23}$ An important finding of our study was that the exercise regimen had no significant effects on synovial fluid 3B3 or 7D4 epitope levels. After acute injuries of the knee, levels of 3B3 and 7D4 in synovial fluid were raised two- to threefold, and tended to be higher in the first three months after injury, and then decline. ${ }^{21}$ This pattern may reflect an early phase of articular cartilage matrix degradation, with clearance of these chains into the synovial fluid, ${ }^{21}{ }^{29}$ and with these markers being less sensitive indicators of chronic cartilage degradation in OA. They were not useful in discriminating between patients with chronic progressive knee OA and those with nonprogressive disease. ${ }^{31}$

This was the first study of temporal changes in the expression of these chondroitin sulphate synovial marker levels, evaluating the effect of exercise. Previous studies of these markers have been on single samples from each patient, and comparisons were made between patient groups. ${ }^{31}{ }^{32}$ The design of our study was statistically more powerful because patients were randomised, studied prospectively, and compared with themselves, thus eliminating the variability associated with between-patient comparisons. Although we observed a trend for the dependent variables to decline with exercise, none of these changes was statistically significant. The possibility exists that lack of significance was due to a type II statistical error, arising from small sample size or large standard deviations of data. ${ }^{33}$ Our power calculations showed that our study was adequately powered for analyses of GAG and 7D4/GAG data, was less so for 3B3 and 7D4 data, and not at all so for the $3 \mathrm{~B} 3 / \mathrm{GAG}$ data.

One problem that evolved with our study was that the control and exercise groups differed in age and BMI at time zero baseline. Although we randomly allocated patients into the two groups at the start of the study, loss of subjects from the study due to inability to collect synovial fluid by arthrocentesis, led to this imbalance. To control for these prior group differences we analysed data using a model that included treatment, time, and treatment-time interaction, as well as subject covariates (sex, age, BMI, and K-L grade). Although we found that GAG levels in the exercise group were significantly lower than in the control group at 12 weeks, the importance of this between-group difference is unknown because of the differences in subject characteristics mentioned above. Levels of GAG in synovial fluid from osteoarthritic joints were previously reported to be lower than in normal joints. ${ }^{32}$ The effect of exercise on synovial fluid GAG has not been studied previously, but this relation may be quite complex and influenced by other factors. For example, we found that the decline in 3B3, GAG, and 3B3/GAG ratio with age, irrespective of treatment, was also significant. This was in contrast with the observation that synovial fluid 3B3 concentration in normal knees was positively correlated with age, in a group of younger subjects. ${ }^{32}$ These associations may relate to a change in metabolic activity of chondrocytes with aging, or perhaps advancing OA, with alterations in the sulphation and length of chondroitin sulphate chains modulating the immunoreactivity of those chains ending with the disaccharide containing the 3B3 epitope..$^{19-21} 29{ }^{34}$ Although we noted a trend for older patients to have higher K-L grades (fig 1 ), this association was not significant. Similarly, other studies of patients with chronic 
knee OA found no relation between synovial fluid markers and K-L scores, scintigraphic scan, or synovial osteocalcin levels. ${ }^{32}{ }^{35}$ This is probably not unexpected, as bone changes do not necessarily occur in unison with cartilage degradation in OA. ${ }^{31}{ }^{35}$

One of the continuing controversies in synovial fluid marker studies is the influence of changes in synovial fluid volumes in joints with effusion, and the consequent dilution of any marker concentration measured. We chose to "normalise" chondroitin sulphate epitope levels against GAG, as has been done in previous studies. ${ }^{31}{ }^{35}$ In conclusion, we found no significant deleterious effects on osteoarthritic joints, as reflected by chondroitin sulphate synovial fluid markers, in patients undergoing an exercise programme that effectively ameliorated joint pain.

1 Dieppe P. Osteoarthritis management. In: Klippel JH, Dieppe PA, eds. Rheumatology. St Louis: Mosby 1994:7.8.1.

2 Felson DT Epidemiology of hip and knee osteoarthritis. Epidemiol Rev 1988;10:1-28.

3 Van Baar ME, Assendelft WJJ, Dekker J, Oostendorp RAB, Bijlsma JWJ. Effectiveness of exercise therapy in patients with osteoarthritis of the hip or knee. Arthritis Rheum $1999 ; 42: 1361-9$

4 O'Reilly SC, Muir KR, Doherty M. Effectiveness of home exercise on pain and disability from osteoarthritis of the knee: a randomized controlled trial. Ann Rheum Dis 1999; 58:15-19.

5 Bautch JC, Malone DG, Vailas AC. Effects of exercise on knee joints with osteoarthritis: a pilot study of biologic markers. Arthritis Care Research 1997; 10:48-55.

6 Kovar PA, Allegrante JP, Mackenzie R, Peterson MGER, Gutin B, Charlson ME. Supervised fitness walking in patients with osteoarthritis of the knee. Ann Intern Med 1992;116:511-15.

7 Minor MA, Hewett JE, Webel, RR, Anderson SK, Kay DR. Efficacy of physical conditioning exercise in patients with rheumatoid arthritis and osteoarthritis. Arthritis Rheum rheumatoid arthritis

8 Jurvelin J, Kiviranta I, Tammi M, Helminen HJ. Effect of prvelin J, Kiviranta I, Tammi M, Helminen HJ. Effect of physical exercise on indentation stiffness of articular carti-
lage in the canine knee. Int J Sports Med 1986;7:106-10.

9 Arokoski J, Kiviranta I, Jurvelin J, Tammi M, Helminen HJ. Long-distance running causes site-dependent decrease of cartilage glycosaminoglycan content in the knee joints of beagle dogs. Arthritis Rheum 1993;36:1451-9.

10 Arokoski J, Jurvelin J, Kiviranta I, Tammi M, Helminen HJ. Softening of the lateral condyle articular cartilage in the canine knee joint after long distance (up to $40 \mathrm{~km} /$ day) running training lasting one year. Int J Sports Med 1994;15:254-60.

11 Saamamen A-M, Kiviranta I, Jurvelin J, Helminen HJ, Tammi M. Proteoglycan and collagen alterations in canine knee articular cartilage following $20 \mathrm{~km}$ daily running exercise for 15 weeks. Connect Tissue Res 1994;30:191-201.

12 Kiviranta I, Tammi M, Jurvelin J, Arokoski J, Saamanen AM, Helminen HJ. Articular cartilage thickness and glycosaminoglycan distribution in the canine knee joint glycosaminoglycan distribution in the canine knee joint
after strenuous running exercise. Clin Orthop 1992;283: 302-8.

13 Little CB, Ghosh P, Rose R. The effect of strenuous versus moderate exercise on the metabolism of proteoglycans in articular cartilage from different weight-bearing regions of the equine third carpal bone. Osteoarthritis Cartilage 1997;5:161-72

14 Spector TD, Harris PA, Hart DJ, Cicuttini FM, Nandra D, Etherington J, et al. Risk of osteoarthritis associated with long-term weight-bearing sports. Arthritis Rheum 1996, 39:988-95
15 Roos H, Dahlerg L, Hoerrner LA, Lark MW, Thonar EJ, Shinmei M, et al. Markers of cartilage matrix metabolism in human joint fluid and serum: the effect of exercise. Osteoarthritis Cartilage 1995;3:7-14.

16 Todhunter RJ, Yeager AE, Freeman KP, Parent EJ, Lust G. Keratan sulfate as a marker of articular cartilage catabolism and joint treatment in ponies. Am J Vet Res 1993;54:100716.

17 Ratcliffe A, Doherty M, Maini RN, Hardingham TE. Increased concentrations of proteoglycan components in synovial fluids of patients with acute but not chronic joint disease. Ann Rheum Dis 1988;47:826-32.

18 Caterson B, Hughes CE, Johnstone B, Mort JS. Immunological markers of cartilage proteoglycan metabolism in animal and human osteoarthritis. In: Kuettner K, ed. Articular cartilage and osteoarthritis. New York: Raven Press 1992:415-27.

19 Caterson B, Hughes CE, Roughley P, Mort JS. Anabolic and catabolic markers of proteoglycan metabolism in osteoarthritis. Acta Orthop Scand Suppl 1995;66 (suppl 266): 121-4.

20 Slater RR, Bayliss MT, Lachiewicz PF, Visco DM, Caterson B. Monoclonal antibodies that detect biochemical marker of arthritis in humans. Arthritis Rheum 1995;38:655-9.

21 Hazell PK, Dent C, Fairclough JA, Bayliss MT, Hardingham TE. Changes in glycosaminoglycan epitope levels in knee joint fluid following injury. Arthritis Rheum 1995; 38:953-9.

22 Osendorf RH, Van de Stadt RJ, Van Kampen GPJ. Intermittent loading induces the expression of 3-B-3(-) epitope in cultured bovine articular cartilage. J Rheumatol 1994;21: 287-92.

23 Ostendorf RH, De Koning MHMT, Van de Stadt RJ, Van Kampen GPJ. Cyclic loading is harmful to articular cartilage from which proteoglycans have been partially depleted by retinoic acid. Osteoarthritis Cartilage 1995;3: 275-84

24 Altman R, Asch E, Bloch D, Bole G, Borenstein D, Brandt $\mathrm{K}$, et al. Development of criteria for the classification and reporting of osteoarthritis. Arthritis Rheum 1986;29:103949.

25 Kellgren JH, Lawrence JS. Radiological assessment of osteo-arthritis. Ann Rheum Dis 1957;16:494-501.

26 NIDDK National task force on prevention and treatment of obesity: executive summary of the clinical guidelines on the identification, evaluation, and treatment of overweight and obesity in adults. Arch Intern Med 1998;158:1855-67.

27 Farndale RW, Buttle DJ, Barrett AJ. Improved quantitation and discrimination of sulphated glycosaminoglycans by use of dimethylmethylene blue. Biochim Biophys Acta 1986; 883:173-7.

28 Plaas AHK, Wong-Palms S, Roughley PJ, Midura RJ, Hascall VC. Chemical and immunological assay of the nonreducing terminal residues of chondroitin sulfate from human aggrecan. J Biol Chem 1997;272:20603-10.

29 Plaas AHK, West LA, Wong-Palms S, Nelson FRT. Glycosaminoglycan sulfation in human osteoarthritis. J Biol Chem 1998;273:12642-9.

30 Carlson CS, Loeser RF, Johnstone B, Tulli HM, Dobson DB, Caterson B. Osteoarthritis in Cynomolgus macaques. II. Detection of modulated proteoglycan epitopes in cartilage and synovial fluid. J Orthop Res 1995;13:399-409.

31 Fawthrop F, Yaqub R, Belcher C, Bayliss M, Ledingham J, Doherty $M$. Chondroitin and keratan sulphate epitopes, glycosaminoglycans, and hyaluronan in progressive versus non-progressive osteoarthritis. Ann Rheum Dis 1997;56: 119-22.

32 Belcher C, Yaqub R, Fawthrop F, Bayliss M, Doherty M. Synovial fluid chondroitin and keratan sulphate epitopes, glycosaminoglycans, and hyaluronan in arthritic and normal knees. Ann Rheum Dis 1997;56:299-307.

33 Markel MD. The power of a statistical test: What does insignificance mean? Vet Surg 1991;20:209-14.

34 Lewis S, Crossman M, Flannelly J, Belcher C, Doherty M, Bayliss MT, et al. Chondroitin sulphation patterns in synovial fluid in osteoarthritis subsets. Ann Rheum Dis 1999;58:441-5.

35 Sharif M, George E, Dieppe PA. Correlation between synovial fluid markers of cartilage and bone turnover and scintigraphic scan abnormalities in osteoarthritis of the knee. Arthritis Rheum 1995;38:78-81. 